\title{
Formation Control and Collision Avoidance for Multi-Agent Systems and a Connection between Formation Infeasibility and Flocking Behavior
}

\author{
Dimos V. Dimarogonas and Kostas J. Kyriakopoulos
}

\begin{abstract}
A feedback control strategy that achieves convergence of a multi-agent system to a desired formation configuration avoiding at the same time collisions is proposed. The collision avoidance objective is handled by a decentralized navigation function that vanishes when the desired formation tends to be realized. When inter-agent objectives that specify the desired formation cannot occur simultaneously in the state space the desired formation is infeasible. It is shown that under certain assumptions, formation infeasibility forces the agents velocity vectors to a common value at steady state. This provides a connection between formation infeasibility and flocking behavior for the multi-agent system.
\end{abstract}

\section{INTRODUCTION}

Multi-agent Navigation is a field that has recently gained increasing attention both in the robotics and the control communities, due to the need for autonomous control of more than one mobile robotic agents in the same workspace. While most efforts in the past had focused on centralized planning, specific real-world applications have lead researchers throughout the globe to turn their attention to decentralized concepts. The motivation for this work comes from many application domains one of the most important of which is the field of micro robotics, ([14],[7]), where a team of a potentially large number of autonomous micro robots must cooperate in the sub micron level.

Among the various specifications that the control design aims to impose on the multi-agent team, formation convergence and achievement of flocking behavior are two objectives that have been pursued extensively in the last few years. The main feature of formation control is the cooperative nature of the equilibria of the system. Agents must converge to a desired configuration encoded by the interagent relative positions. Many feedback control schemes that achieve formation stabilization to a desire formation in a distributed manner have been proposed in literature, see for example [20],[12],[11],[3] for some recent efforts. Of particular interest is also the so-called agreement problem, in which agents must converge to the same point in the state space ([15], [18],[2],[9]). On the other hand, flocking behavior involves convergence of the velocity vectors and orientations of the agents to a common value at steady state; contributions include [8], [19],[17].

\footnotetext{
The authors are with the Control Systems Lab, Department of Mechanical Engineering, National Technical University of Athens, 9 Heroon Polytechniou Street, Zografou 15780, Greece ddimar,kkyria@mail.ntua.gr
}

In this paper, the problem of formation control is considered. The main feature of formation control is the cooperative nature of the equilibria of the system. Agents must converge to a desired configuration encoded by the inter-agent relative positions. Inspired by our previous work ([3],[5]) involving decentralized navigation and collision avoidance of multiagent systems to non-cooperative equilibria (i.e. each agent had a specific goal configuration not related to the positions of the others) in this paper we propose a methodology that handles the problem of formation control satisfying at the same time, the collision avoidance objective for the sphere world case. We must note that the same problem has been considered in [20] for the point world case. In this paper, we exploit our previous results to treat the sphere world case.

In most cases, formation convergence involves kinematic models of the agents' motion, while flocking behavior dynamic ones. Hence the problem of flocking motion has rarely been examined in the context of kinematic models of motion. In this paper, a connection between formation infeasibility and flocking behavior for multiple kinematic agents is established. Formation infeasibility is equivalent to the case when inter-agent objectives cannot occur simultaneously in the state space. By decoupling the two objectives (collision avoidance and formation convergence) it is shown that under certain assumptions formation infeasibility forces the agents velocity vectors to a common value at steady state.

The rest of the paper is organized as follows: section II presents the system definition and problem statement. Section III presents the proposed control scheme. The stability analysis is provided in section IV. Section V contains an interesting result relating formation infeasibility and flocking behavior. In section VI computer simulation results are presented while section VII summarizes the conclusions and indicates our current research.

\section{System AND PRoblem Definition}

Consider a system of $N$ spherical agents operating in the same workspace $W \subset \mathcal{R}^{2}$. Each agent $i$ occupies a disc: $R=$ $\left\{q \in \mathcal{R}^{2}:\left\|q-q_{i}\right\| \leq r_{i}\right\}$ in the workspace where $q_{i} \in \mathcal{R}^{2}$ is the center of the disc and $r_{i}$ is the radius of the agent. The configuration space is spanned by $q=\left[q_{1}, \ldots, q_{N}\right]^{T}$. The motion of each agent is described by the single integrator:

$$
\dot{q}_{i}=u_{i}, i \in \mathcal{N}=[1, \ldots, N]
$$

where $u_{i}$ denotes the velocity (control input) for each agent. Each agents' objective is to converge to a desired relative 
configuration with respect to a certain subset of the rest of the team, in a manner that will lead the whole team to a desired formation. Specifically, each agent is assigned with a specific subset $N_{i}$ of the rest of the team, called agent $i$ 's neighboring set with which it can communicate in order to achieve the desired formation. Following the literature on formation control [16],[19], the desired formation can be encoded in terms of a formation graph:

Definition 1: The formation graph $G=\{V, E, C\}$ is an undirected graph that consists of (i) a set of vertices $V=$ $\{1, \ldots, N\}$ indexed by the team members, (ii) a set of edges, $E=\left\{(i, j) \in V \times V \mid i \in N_{j}\right\}$ containing pairs of nodes that represent inter-agent formation specifications and (iii) a set of labels $C=\left\{c_{i j}\right\}$, where $(i, j) \in E$, that specify the desired inter-agent relative positions in the formation configuration.

The objective of each agent $i$ is to be stabilized in a desired relative position $c_{i j}$ with respect to each member $j$ of $N_{i}$, avoiding at the same time collisions.

Collision avoidance is meant in the sense that no intersections occur between the agents' discs. Thus we want to assure that $\left\|q_{i}(t)-q_{j}(t)\right\|>r_{i}+r_{j}, \forall i, j \in \mathcal{N}, i \neq j$ for each time instant $t$. This is a key difference of collision avoidance of non-point agents with respect to point agents. In the latter case, collisions (in the two dimensional world) occur only when $\left\|q_{i}(t)-q_{j}(t)\right\|=0$ for some $i, j$. This is not the case for the non-point world as can be seen by the previous equation. Thus a different machinery is used in the non-point case. In previous work [5],[13],[3] we used the navigation functions approach, established by Koditschek and Rimon in the seminal paper [10], to achieve collision avoidance and destination convergence for multiple spherical agents. In this paper the destination convergence objective is replaced by formation convergence.

Hence, the problem treated in this paper can be stated as follows: "derive a set of control laws (one for each agent) that drives the team of agents from any initial configuration to the desired formation configuration avoiding, at the same time, collisions.".

The following assumptions highlight the level of decentralization of the approach:

1) Each agent has only knowledge of the position of agents located in a cyclic neighborhood of specific radius $d_{C}$ at each time instant, where $d_{C}>$ $\max _{i, j \in \mathcal{N}}\left(r_{i}+r_{j}\right)$. This set $S_{i}=\left\{q:\left\|q-q_{i}\right\| \leq d_{C}\right\}$ is called the sensing zone of agent $i$. Hence apart from knowledge of agent located in $N_{i}, i$ has also knowledge of the positions of agents in $S_{i}$.

2) Each agent knows the exact number of agents in the workspace.

3) The workspace is bounded and spherical. Specifically $W=\left\{q:\|q\| \leq R_{w}\right\}$, where $R_{w}$ denotes the workspace radius.

4) The formation graph is undirected, in the sense that $i \in N_{j} \Leftrightarrow j \in N_{i}, \forall i, j \in \mathcal{N}, i \neq j$. It is obvious that $(i, j) \in E$ iff $i \in N_{j} \Leftrightarrow j \in N_{i}$.

5) There are no conflicting inter-agent objectives, in the sense that $c_{i j}=-c_{j i}, \forall i, j \in \mathcal{N}, i \neq j$.

6) The formation configuration is feasible, in the sense that $\exists q:\left\|q_{i}-q_{j}-c_{i j}\right\|=0, \forall(i, j) \in E$. The set $E_{q}=\left\{q:\left\|q_{i}-q_{j}-c_{i j}\right\|=0 \forall(i, j) \in E\right\}$ is called the equilibrium set of the formation.

The next figure shows two examples of feasible formation configurations in a team of four and seven agents respectively, as well as the corresponding neighboring sets for each agent in the second case. The line formation configuration of the second figure is implemented in the simulation section. It is obvious that the radii of the agents do not have to be equal.

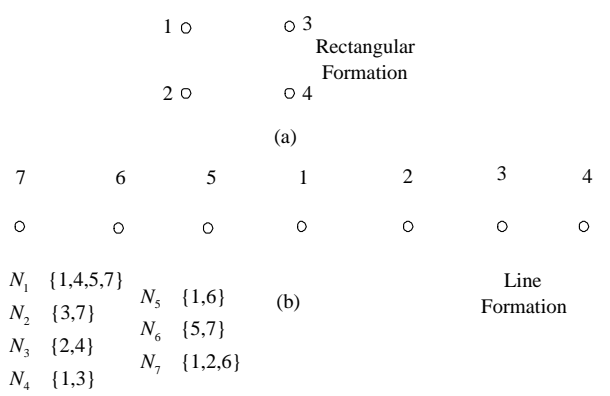

Fig. 1. Feasible formation configuration example of (a) a rectangular and (b)a line formation

\section{Control Strategy}

The proposed feedback control strategy for agent $i$ is defined as

$$
u_{i}=-K_{i} \frac{\partial \varphi_{i}}{\partial q_{i}}-D_{i} \frac{\partial \gamma_{i}}{\partial q_{i}}
$$

where $K_{i}, D_{i}$ are positive gains.

The function $\gamma_{i}: W \rightarrow \mathcal{R}_{+}$represents the control objective for agent $i$ : converging to a desired relative configuration with respect to each $j \in N_{i}$. A suitable choice is:

$$
\gamma_{i}=\frac{1}{2} \sum_{j \in N_{i}}\left\|q_{i}-q_{j}-c_{i j}\right\|^{2}
$$

Function $\varphi_{i}$ is a navigation function that ensures collision avoidance between agents in the team. Inspired by our previous work on decentralized navigation functions ([5],[3]), $\varphi_{i}$ is constructed to assure collision avoidance between the agents in a decentralized manner:

$$
\varphi_{i}=\frac{f_{i}\left(G_{i}\right)}{\left(\left(f_{i}\left(G_{i}\right)\right)^{k}+G_{i}\right)^{1 / k}}
$$

The function $G_{i}$ serves as an encoder of all possible collision schemes between agent $i$ and the rest of the team. It is designed in such a way to ensure that the boundary of the free space of each agent is repulsive with respect to the produced gradient motion. The free space for each agent is defined as the subset of $W$ which is free of collisions with the other agents. Collision avoidance is reassured in a bounded workspace and for appropriate tuning of the controller gains. Under the assumptions of the previous section, $G_{i}$ is defined 
so that each agent takes into account the positions of agents that are within its sensing zone at each time instant. However, decentralization is restricted by the fact that the construction of $G_{i}$ requires knowledge of the exact number of agents in the state space. The parameter $k$ is a positive constant which as shall be shown in the sequel must be sufficiently large to guarantee system stability. The constructive procedure to define $G_{i}$ and more details can be found in [3](see also [4] for the global sensing case).

Function $f_{i}$ is defined in such a way to ensure that the repulsive potential vanishes when inter-agent distances are sufficiently large. This function has also been used in our previous work ([5],[3]) and was introduced in [21]. We define the function $f_{i}$ by:

$$
f_{i}\left(G_{i}\right)=\left\{\begin{array}{l}
a_{0}+\sum_{j=1}^{3} a_{j} G_{i}^{j}, G_{i} \leq X \\
0, G_{i}>X
\end{array}\right.
$$

where $X, Y=f_{i}(0)>0$ are positive scalar constants. The parameters $a_{j}$ are evaluated so that $f_{i}$ is maximized when $G_{i} \rightarrow 0$ and minimized when $G_{i}=X$. We also require that $f_{i}$ is continuously differentiable at $X$. Therefore we have: $a_{0}=Y, a_{1}=0, a_{2}=\frac{-3 Y}{X^{2}}, a_{3}=\frac{2 Y}{X^{3}}$. The parameter $X$ serves as a sensing parameter that activates the $f_{i}$ function whenever possible collisions are bound to occur. The only requirement we have for $X$ is that it must be small enough to guarantee that $f_{i}$ vanishes whenever the system has reached its equilibrium set, i.e. when $q \in S$. In mathematical terms:

$$
X<G_{i}(q), \forall q \in E_{q}, \forall i
$$

This constraint ensures that the repulsive potential vanishes at the formation configurations.

\section{Stability AnAlysis}

\section{A. Tools from Algebraic Graph Theory}

In this subsection we review some tools from algebraic graph theory that we shall use in the stability analysis the next sections. The following can be found in any standard textbook on algebraic graph theory(e.g. [1]).

For an undirected graph $G$ with $n$ vertices the adjacency matrix $A=A(G)=\left(a_{i j}\right)$ is the $n \times n$ matrix given by $a_{i j}=1$ if $(i, j) \in E$ and $a_{i j}=0$ otherwise. The degree $d_{i}$ of vertex $i$ is defined as the number of its neighboring vertices, i.e. $d_{i}=\{\# j:(i, j) \in E\}$. Let $\Delta$ be the $n \times n$ diagonal matrix of $d_{i}$ 's. The (combinatorial) Laplacian of $G$ is the symmetric positive semidefinite matrix $\mathcal{L}=\Delta-A$. The Laplacian captures many interesting topological properties of the graph. Of particular interest in our case is the fact that for a connected graph, the Laplacian has a single zero eigenvalue and the corresponding eigenvector is the vector of ones, $\overrightarrow{\mathbf{1}}$.

The last property has lead to the interesting result regarding the connection between formation non-feasibility and flocking behavior discussed in section V. The next subsection contains the stability analysis of the formation scheme.

\section{B. Stability of a feasible formation}

Convergence of the agents to the desired formation configuration is guaranteed by the following theorem:

Theorem 1: Assume that the following hold:

- The equilibrium set in nonempty, i.e. $E_{q} \neq \emptyset$.

- $X$ is small enough to guarantee that if $\exists i: G_{i}<X$ then

$$
\exists \delta>0:\left\|\left(\mathcal{L} \otimes I_{2}\right) q+c_{l}\right\| \geq \delta
$$

where $\mathcal{L}$ is the Laplacian of the formation graph, the vector $c_{l}$ is defined by $c_{l}=\left[c_{11}, \ldots, c_{N N}\right]^{T}$, with $c_{i i}=$ $-\sum_{j \in N_{i}} c_{i j}$ and where $\otimes$ denotes Kronecker product.

Then, under the feedback control strategy (2), the state of the system converges to $E_{q}$, provided that $k$ is bounded from below by a finite lower bound.

Proof: Function $V=\sum_{i}\left(\varphi_{i}+\gamma_{i}\right)$ is used as a candidate Lyapunov function for the whole system. Taking its derivative we have

$$
V=\sum_{i}\left(\varphi_{i}+\gamma_{i}\right) \Rightarrow \dot{V}=\left\{\sum_{i}\left(\nabla \varphi_{i}+\nabla \gamma_{i}\right)^{T}\right\} \cdot \dot{q}
$$

Remembering that $u_{i}=-K_{i} \frac{\partial \varphi_{i}}{\partial q_{i}}-D_{i} \frac{\partial \gamma_{i}}{\partial q_{i}}$ and that $\varphi_{i}=$ $\frac{f_{i}\left(G_{i}\right)}{\left(\left(f_{i}\left(G_{i}\right)\right)^{k}+G_{i}\right)^{1 / k}}$ the closed loop dynamics of the system are given by:

$$
\begin{aligned}
& \dot{q}=\left[\begin{array}{l}
-K_{1} A_{1}^{-(1+1 / k)} \sigma_{1} \frac{\partial G_{1}}{\partial q_{1}}-D_{1} \frac{\partial \gamma_{1}}{\partial q_{1}} \\
\vdots \\
-K_{N} A_{N}^{-(1+1 / k)} \sigma_{N} \frac{\partial G_{N}}{\partial q_{N}}-D_{N} \frac{\partial \gamma_{N}}{\partial q_{N}}
\end{array}\right]=\ldots \\
& =-A_{K} \Sigma Q q-D\left(L q+c_{l}\right)
\end{aligned}
$$

where $\sigma_{i}=G_{i} \sigma\left(G_{i}\right)-\frac{f_{i}}{k}, A_{i}=f_{i}^{k}+G_{i}, \sigma\left(G_{i}\right)=$ $\sum_{j=1}^{3} j a_{j} G_{i}^{j-1}$ and the matrices

$$
\begin{gathered}
A_{K} \triangleq \underbrace{\operatorname{diag}\left(\begin{array}{c}
K_{1} A_{1}^{-(1+1 / k)}, K_{1} A_{1}^{-(1+1 / k)}, \ldots, \\
K_{N} A_{N}^{-(1+1 / k)}, K_{N} A_{N}^{-(1+1 / k)}
\end{array}\right)}_{2 N \times 2 N} \\
D \triangleq \underbrace{\operatorname{diag}\left(D_{1}, D_{1}, \ldots, D_{N}, D_{N}\right)}_{2 N \times 2 N}
\end{gathered}
$$

$\Sigma \triangleq[\underbrace{\Sigma_{1}}_{2 N \times 2 N}, \ldots, \underbrace{\Sigma_{N}}_{2 N \times 2 N}], \Sigma_{i}=\operatorname{diag}\left(\begin{array}{c}0, \ldots, \underbrace{\sigma_{i}, \sigma_{i}}_{2 i-1,2 i}, \\ \ldots, 0\end{array}\right)$

Without loss of generality, we assume that $D_{i}=D$ for all $i$. We will use interchangeably the notation $D$ both for the matrix $D$ as well as for its equal elements. The matrix $Q$ is defined by the following relation:

$$
\nabla G \triangleq\left[\begin{array}{l}
\nabla G_{1} \\
\vdots \\
\nabla G_{N}
\end{array}\right]=\left[\begin{array}{l}
Q^{1} \\
\vdots \\
Q^{N}
\end{array}\right] q \triangleq Q q
$$


Analytic expressions for the elements of the matrices $Q^{i}$ can be found in [4]. Each $Q_{i}$ is symmetric, i.e. $Q_{i}=Q_{i}^{T}$. We also have $Q_{i j}^{i}=Q_{j i}^{i}=-Q_{j j}^{i}$ and $Q_{j k}^{i}$ whenever $i \neq j \neq k \neq i$.

The matrix $L$ corresponds to the desired inter-agent relative positions and can be shown to be related with the Laplacian of the formation graph by the relation $L=\mathcal{L} \otimes I_{2}$. That's a crucial result regarding the behavior of the system as we shall we in the next section.

The gradient of $\sum_{i} \gamma_{i}$ can be calculated by

$$
\sum_{i}\left(\nabla \gamma_{i}\right)^{T}=\ldots=2\left(q^{T} L+c_{l}^{T}\right)
$$

Similarly, the gradient of $\sum_{i} \varphi_{i}$ is calculated by $\sum_{i}\left(\nabla \varphi_{i}\right)^{T}=\ldots=q^{T} Q^{T} A_{\Sigma}$, where

$$
A_{\Sigma}=\left[\begin{array}{l}
A_{\Sigma_{1}} \\
\vdots \\
A_{\Sigma_{N}}
\end{array}\right], A_{\Sigma_{i}}=\underbrace{\operatorname{diag}\left(\begin{array}{c}
A_{i}^{-(1+1 / k)} \sigma_{i}, \ldots, \\
A_{i}^{-(1+1 / k)} \sigma_{i}
\end{array}\right)}_{2 N \times 2 N}
$$

By using the notation $z \triangleq L q+c_{l}$, the derivative of the candidate Lyapunov function is now calculated as

$$
\begin{aligned}
& \dot{V}=\left\{\sum_{i}\left(\nabla \varphi_{i}+\nabla \gamma_{i}\right)^{T}\right\} \cdot \dot{q}=\ldots \\
& =-D\|z\|^{2}-\left[\begin{array}{ll}
z^{T} & q^{T}
\end{array}\right] M\left[\begin{array}{c}
z \\
q
\end{array}\right]
\end{aligned}
$$

where $M \triangleq\left[\begin{array}{cc}D & 2 A_{K} \Sigma Q \\ Q^{T} A_{\Sigma} D & Q^{T} A_{\Sigma} A_{K} \Sigma Q\end{array}\right]$. Hence

$$
\dot{V} \leq-D\|z\|^{2}+\left|\lambda_{\min }(H(M))\right|\left(\|z\|^{2}+\|q\|^{2}\right)
$$

where $H(M)=\frac{1}{2}\left(M+M^{T}\right)$ the Hermitian part of the matrix $M$ and $\lambda_{\min }(H(M))$ its largest negative eigenvalue. The positive definiteness of the matrix $M$ cannot be guaranteed due to the fact that each agent has to take into account in the conflict resolution procedure agents that do not belong in its neighboring set. The Hermitian part of the matrix $M$ is $H(M)=\frac{1}{2}\left[\begin{array}{ll}H_{1} & H_{2} \\ H_{3} & H_{4}\end{array}\right]$, where $H_{1}=2 D, H_{2}=$ $2 A_{K} \Sigma Q+D A_{\Sigma}^{T} Q, H_{3}=Q^{T} A_{\Sigma} D+2 Q^{T} \Sigma^{T} A_{K}, H_{4}=$ $Q^{T} A_{\Sigma} A_{K} \Sigma Q+Q^{T} \Sigma^{T} A_{K} A_{\Sigma}^{T} Q$. After some calculation, we have

$$
\begin{aligned}
& \left(H_{2}\right)_{i j}=\left(H_{3}\right)_{i j}= \\
& =\left\{\begin{array}{l}
2 K_{i} \sigma_{i} A_{i}^{-(1+1 / k)} Q_{i i}^{i}+\sum_{l} D A_{l}^{-(1+1 / k)} \sigma_{l} Q_{i i}^{l}, i=j \\
\sum_{l} D A_{l}^{-(1+1 / k)} \sigma_{l} Q_{i j}^{l}, i \neq j
\end{array}\right. \\
& \left(H_{4}\right)_{i j}=\sum_{l}\left(A_{l} A_{j}\right)^{-(1+1 / k)} \sigma_{l} \sigma_{j} K_{j} Q_{i j}^{l} Q_{j j}^{j}
\end{aligned}
$$

and

$$
+\sum_{l}\left(A_{l} A_{i}\right)^{-(1+1 / k)} \sigma_{l} \sigma_{i} K_{i} Q_{i i}^{i} Q_{i j}^{l}
$$

Specific bounds on each of the terms in the last relations are provided in [4]. In particular in [4] it is shown that the term $\sigma_{i}$ is always negative and bounded and its bounds are given by $0 \leq\left|\sigma_{i}\right| \leq \frac{\Theta}{k}$ where $\Theta$ is a scalar positive parameter.
This relation, along with the fact that the $\sigma_{i}$ terms are present in the analytic expressions of $\left(H_{k}\right)_{i j}, k=2,3,4$ helps us in deriving bounds on $k$ that ensure asymptotic stability.

In the following analysis we make use of the following theorems from matrix analysis ([6]) to provide an estimate of $\lambda_{\min }(H(M))$ :

Theorem 4.1: Given a matrix $A \in R^{n \times n}$ then all its eigenvalues lie in the union of $n$ discs:

$$
\bigcup_{i=1}^{n}\left\{z:\left|z-a_{i i}\right| \leq \sum_{\substack{j=1 \\ j \neq i}}^{n}\left|a_{i j}\right|\right\} \triangleq \bigcup_{i=1}^{n} R_{i}(A) \triangleq R(A)
$$

Each of these discs is called a Gersgorin disc of A.

Corollary 4.2: Given a matrix $A \in R^{n \times n}$ and $n$ positive real numbers $p_{1}, \ldots, p_{n}$ then all the eigenvalues of $\mathrm{A}$ lie in the union of $n$ discs:

$$
\bigcup_{i=1}^{n}\left\{z:\left|z-a_{i i}\right| \leq \frac{1}{p_{i}} \sum_{\substack{j=1 \\ j \neq i}}^{n} p_{j}\left|a_{i j}\right|\right\}
$$

By using $p_{1}=\ldots=p_{2 N}=p$ for the first $2 N$ rows of $H(M)$, Corollary 4.2 provides the following estimates for the eigenvalues corresponding to these rows:

$$
|z-2 D| \leq \sum_{j=1}^{2 N} \frac{p_{2 N+j}}{p}\left|\left(H_{2}\right)_{i j}\right| \leq \frac{2 N}{p} \max _{j} p_{2 N+j}\left|\left(H_{2}\right)_{i j}\right|
$$

The form of $\left(H_{2}\right)_{i j}$ guarantees that $\max _{j} p_{2 N+j}\left|\left(H_{2}\right)_{i j}\right| \leq$ $\frac{\Theta_{2}}{k}$, where $\Theta_{2}$ a positive finite bound that corresponds to the upper bounds of the terms $A_{i}^{-(\cdot)},\left|Q_{i j}^{l}\right|, K_{i}$. The fact that these bounds are finite is discussed in [4]. $\Theta_{2}$ can be calculated explicitly after a series of maximizations on the terms involved in $\left|\left(H_{2}\right)_{i j}\right|$. The fact that the exponent $k$ appears in the denominator is a direct consequence of the fact that a term of the form $\sigma_{i}$ is present in every byproduct of $\left|\left(H_{2}\right)_{i j}\right|$. The corresponding eigenvalues of the matrix $H(M)$ can be rendered strictly positive by tuning $k$ large enough as shown in the following:

$$
|z-2 D| \leq \frac{2 N}{p} \frac{\Theta_{2}}{k} \Rightarrow z>2 D-\frac{2 N}{p} \frac{\Theta_{2}}{k}
$$

Then $z>0$ is guaranteed by $k>\frac{N}{p} \frac{\Theta_{2}}{D}$. Notice that there is no restriction on how to choose the parameters $p_{2 N+j}$ of the last $2 N$ rows, provided that they are finite.

Repeating the procedure for the submatrices $\mathrm{H}_{3}, \mathrm{H}_{4}$ does not guarantee positive definiteness because a careful examination of the analytic forms of $\left(H_{3}\right)_{i j},\left(H_{4}\right)_{i j}$ reveals that some elements of the main diagonal of $H_{4}$ could be zero while some corresponding elements of the $i$-th row corresponding to $H_{3}$ can be nonzero. Hence the Gersgorin discs may intersect with the left half plane of the imaginary axis. However, the following procedure shows that the largest negative eigenvalue can be rendered sufficiently small to guarantee negative definiteness of $\dot{V}$. 
In the worst case , the eigenvalues of rows $2 N+1, \ldots, 4 N$ of the matrix $H(M)$ lie in the disc

$$
|z|<\max _{i} \frac{p}{p_{2 N+i}}\left\{\sum_{j}\left|\left(H_{3}\right)_{i j}\right|+\sum_{j \neq i}\left|\left(H_{4}\right)_{i j}\right|\right\}
$$

Assuming without loss of generality that $p_{2 N+i}=\tilde{p} \forall i=$ $1, \ldots, 2 N$ and using the same logic as above it is straightforward to see that there exists a finite $\Theta_{3}>0$ such that

$$
\max _{i} \frac{p}{p_{2 N+i}}\left\{\sum_{j}\left|\left(H_{3}\right)_{i j}\right|+\sum_{j \neq i}\left|\left(H_{4}\right)_{i j}\right|\right\} \leq \frac{p}{\tilde{p}} \frac{\Theta_{3}}{k}
$$

Hence $\lambda_{\min }(H(M))$ is bounded by $\left|\lambda_{\min }(H(M))\right| \leq \frac{p}{\tilde{p}} \frac{\Theta_{3}}{k}$.

Note that the term $\frac{p}{\tilde{p}}$ can be chosen arbitrarily small, while $\Theta_{3}$ is always bounded in a bounded workspace. Hence

$$
\dot{V} \leq-D\|z\|^{2}+\frac{p}{\tilde{p}} \frac{\Theta_{3}}{k}\left(\|z\|^{2}+\|q\|^{2}\right)
$$

The latter is guaranteed to be negative if $k$ is chosen large enough:

$$
\begin{aligned}
& \dot{V}<0 \Leftrightarrow D\|z\|^{2}>\frac{p}{\tilde{p}} \frac{\Theta_{3}}{k}\left(\|z\|^{2}+\|q\|^{2}\right) \Leftrightarrow \\
& \Leftrightarrow k>\frac{p}{\tilde{p}} \frac{\Theta_{3}}{D}\left(1+\frac{R_{w}^{2}}{\delta^{2}}\right)
\end{aligned}
$$

By virtue of the last relation we have that $\dot{V}<0$ if $G_{i}<$ $X$ for some $i$. In the set $G_{i} \geq X \forall i$ we have

$$
\dot{V}=-2 D\|z\|^{2}=-2 D\left\|L q+c_{l}\right\|^{2} \leq 0
$$

Application of LaSalle's invariance principle ensures the convergence of the system to the largest invariant subset of the set $S=\left\{q: L q+c_{l}=0\right\}$ which corresponds to the desired formation configuration. $\diamond$

Note that the sets $S=\left\{q: L q+c_{l}=0\right\}, q: X<$ $G_{i}(q) \forall i$ always intersect due to the constraint (6), provided that the equilibrium set is non-empty.

It is obvious that the second assumption of the theorem statement guarantees the finiteness of the bound on $k$ that leads to asymptotic stability. However it is not as restrictive as it seems since $X$ can be chosen small enough to ensure the validity of the assumption.

\section{FORMATION NON-FEASIBILITY RESULTS IN FLOCKING BEHAVIOR}

The key assumption behind the stability analysis of the previous section is formation feasibility, namely that there exists a configuration $q \in W$ such that $L q+c_{l}=0$. But what happens when there does not exist such a configuration in the state space? The answer is contained in the next theorem:

Theorem 2: Assume that the following hold:

1) $\min _{q \in W}\left\|L q+c_{l}\right\|>0$.

2) $X<G_{i}\left(q^{*}\right), \forall q^{*}:\left\|L q^{*}+c_{l}\right\|=\min _{q \in W}\left\|L q+c_{l}\right\|>0$

3) The formation graph is connected.

Under these assumptions, the system reaches a configuration in which all agents have the same velocities and orientations.

Proof: Equation (8) guarantees that the system converges to a configuration than minimizes $\left\|L q+c_{l}\right\|$. Since
$\left\|L q+c_{l}\right\|_{\min }>0$ we have $L q+c_{l}=c$ at steady state, where $c$ is a constant nonzero vector. Hence $L \dot{q}=0$ at steady state. Assumption 2 and eq.(7) guarantee that at steady state the dynamics of the system are given by $\dot{q}=-D\left(L q+c_{l}\right) \neq 0$. Using the notation $v_{x}, v_{y}$ for the $n$-dimensional stack vectors of the components of the agents' velocities in the $x, y$ directions at steady state, we have

$$
L \dot{q}=0 \Rightarrow \dot{q}^{T}\left(\mathcal{L} \otimes I_{2}\right) \dot{q}=0 \Rightarrow v_{x}^{T} \mathcal{L} v_{x}+v_{y}^{T} \mathcal{L} v_{y}=0
$$

The last relation and the fact that $\dot{q} \neq 0$ guarantees that at least one of the vectors $v_{x}, v_{y}$ is nonzero. Hence at least one of the vectors $v_{x}, v_{y}$ is an eigenvector of $\mathcal{L}$ corresponding to the zero eigenvalue. For a connected graph, the eigenvector associated with the single zero eigenvalue of the Laplacian is the vector of ones, $\overrightarrow{\mathbf{1}}$. Hence at steady state, at least one of the vectors $v_{x}, v_{y}$ belongs in $\operatorname{span}\{\overrightarrow{\mathbf{1}}\}$, which ensures that all agent velocity vectors will have the same components at steady state $\diamond$

This simple result shows that formation non-feasibility is directly related to a phenomenon with many similarities to what is known as flocking behavior in multi-agent systems. The interagent relative positions at steady state as well as the value of the velocity norms are captured by $\min _{q \in W}\left\|L q+c_{l}\right\|$. It is obvious that the form of $c_{l}, L$ is directly related to the form of the resulting flock. The exact hidden relation is quite interesting and is a topic of current research.

\section{Simulations}

To verify the results of the previous paragraphs we provide two nontrivial computer simulations.

The first simulation involves convergence to a feasible formation configuration. Specifically, we implement the line formation of figure 1(b). The neighboring sets of each agent are defined on that picture. Screenshots I-VI show the evolution in time of the multi agent team. The values of the parameters in this simulation are: $k=90, D=0.1 K=10^{-4}, X=Y=$ $10^{-3}$ and $d_{C}=2 \max _{i, j=\{1, \ldots, 7\}}\left(r_{i}+r_{j}\right)=0.09$. The collision avoidance as well as formation configuration properties are both verified. Note that $X$ has been chosen small enough to guarantee that at the equilibrium configuration the condition $G_{i}>X \forall i$ holds even if the agents are very close to each other as witnessed in screenshot VI.

The second simulation involves four agents and a nonfeasible formation configuration. The values of the parameters in this simulation are the same as previously. The neighboring sets and desired inter-agents relative position vectors are $N_{1}=\{2,3,4\}, N_{2}=\{1,4\}, N_{3}=\{1\}, N_{4}=\{1,2\}$, $c_{12}=c_{41}=c_{24}=\left[\begin{array}{ll}-.1 & 0\end{array}\right]^{T}, c_{13}=\left[\begin{array}{ll}0 & -.1\end{array}\right]^{T}$ It can easily be seen that this is not a feasible formation configuration and that the formation graph is connected. Screenshots I-V show the evolution in time of the multi agent team. As can be seen in this figure, the interagent velocities vectors are stabilized at steady state to a common value. This is shown also in the velocity diagram of the last screenshot. 

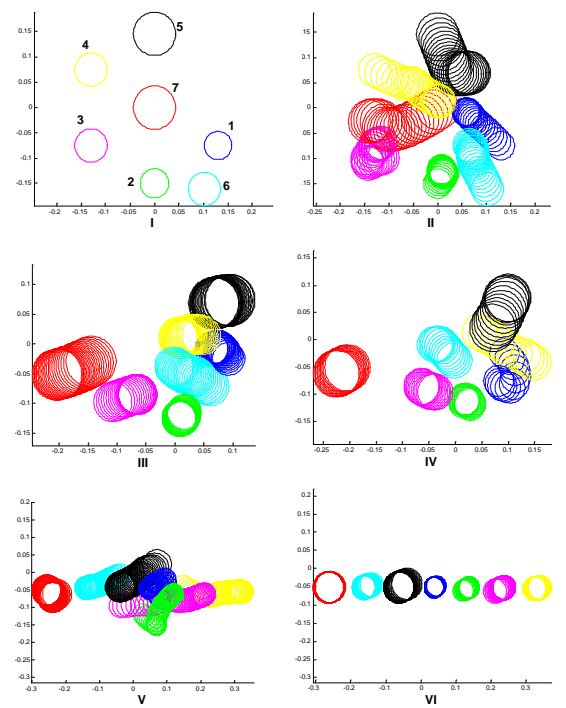

Fig. 2. Seven agents converging to a line formation
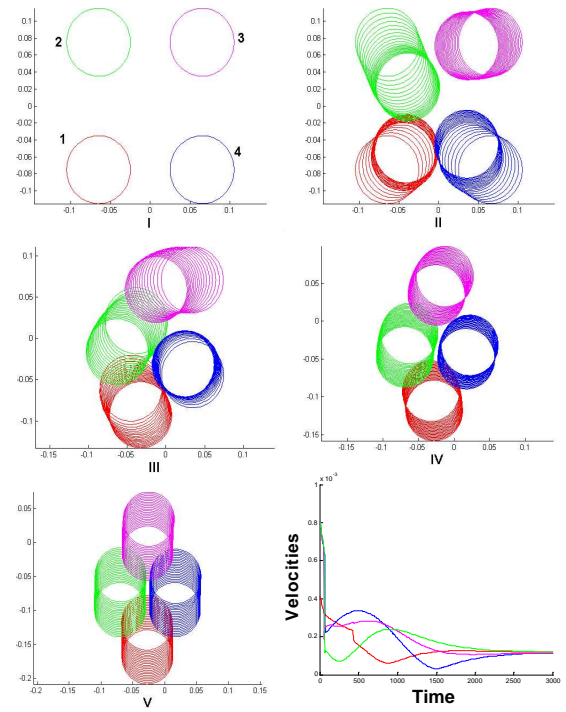

Fig. 3. Flocking behavior for 4 agents. Agents velocities converge to a common value

\section{CONCLUSIONS}

A feedback control strategy that achieves convergence of a multi-agent system to a desired formation configuration avoiding at the same time collisions was proposed. The collision avoidance objective is handled by a decentralized navigation function that vanishes when the desired formation tends to be realized. When inter-agent objectives that specify the desired formation cannot occur simultaneously in the state space the desired formation is infeasible. It was shown that under certain assumptions, formation infeasibility forces the agents velocity vectors to a common value at steady state. This provides a connection between formation infeasibility and flocking behavior for the multi-agent system.

Current research involves further studying the connection between the Laplacian of the formation graph and of the inter-agent desired positions' vector $c_{l}$ with the resulting flock in the case of formation infeasibility as well as building a similar control scheme for formation convergence of nonholonomic agents.

\section{ACKNOWLEDGEMENTS}

The authors want to acknowledge the contribution of the European Commission through contracts MICRON(IST2001-33567) and I-SWARM (IST-2004-507006).

\section{REFERENCES}

[1] B. Bollobás. Modern Graph Theory. Springer Graduate Texts in Mathematics \# 184, 1998.

[2] J. Cortes, S. Martinez, and F. Bullo. Robust rendezvous for mobile autonomous agents via proximity graphs in d dimansions. IEEE Transactions on Automatic Control, submitted for publication, 2004.

[3] D. V. Dimarogonas and K. J. Kyriakopoulos. Decentralized stabilization and collision avoidance of multiple air vehicles with limited sensing capabilities. 2005 American Control Conference, pages 4667 4772

[4] D. V. Dimarogonas, S. G. Loizou, K.J. Kyriakopoulos, and M. M. Zavlanos. A feedback stabilization and collision avoidance scheme for multiple independent non-point agents. Automatica, to appear, 2005.

[5] D.V. Dimarogonas, M.M. Zavlanos, S.G. Loizou, and K.J. Kyriakopoulos. Decentralized motion control of multiple holonomic agents under input constraints. 42nd IEEE Conference on Decision and Control, pages 3390-3395, 2003.

[6] R. A. Horn and C. R. Johnson. Matrix Analysis. Cambridge University Press, 1996.

[7] Project ISWARM. http://microrobotics.ira.uka.de/

[8] A. Jadbabaie, J. Lin, and A.S. Morse. Coordination of groups of mobile autonomous agents using nearest neighbor rules. IEEE Transactions on Automatic Control, 48(6):988-1001, 2003.

[9] M. Ji and M. Egerstedt. Connectedness preserving distibuted coordination control over dynamic graphs. 2005 American Control Conference, pages 93-98.

[10] D. E. Koditschek and E. Rimon. Robot navigation functions on manifolds with boundary. Advances Appl. Math., 11:412-442, 1990.

[11] G. Lafferriere, A. Williams, J. Caughman, and J.J.P. Veerman. Decentralized control of vehicle formations. Systems and Control Letters, 54(9):899-910, 2005.

[12] Z. Lin, B. Francis, and M. Maggiore. Necessary and sufficient graphical conditions for formation control of unicycles. IEEE Transactions on Automatic Control, 50(1):121-127, 2005.

[13] S. G. Loizou and K. J. Kyriakopoulos. Closed loop navigation for multiple holonomic vehicles. Proc. of IEEE/RSJ Int. Conf. on Intelligent Robots and Systems, pages 2861-2866, 2002.

[14] Project MICRON. http://wwwipr.ira.uka.de/ micron/.

[15] L. Moreau. Stability of continuous-time distributed consensus algorithms. 43rd IEEE Conf. Decision and Control, pages 3998-4003, 2004

[16] A. Muhammad and M. Egerstedt. Connectivity graphs as models of local interactions. 43rd IEEE Conf. Decision and Control, pages 124 129, 2004.

[17] R. Olfati-Saber and R.M. Murray. Flocking with obstacle avoidance: Cooperation with limited communication in mobile networks. $42 \mathrm{st}$ IEEE Conf. Decision and Control, pages 2022-2028, 2003.

[18] R. Olfati-Saber and R.M. Murray. Consensus problems in networks of agents with switching topology and time-delays. IEEE Transactions on Automatic Control, 49(9):1520-1533, 2004.

[19] H.G. Tanner, A. Jadbabaie, and G.J. Pappas. Flocking in fixed and switching networks. IEEE Transactions on Automatic Control, submitted for publication, 2005.

[20] H.G. Tanner and A. Kumar. Formation stabilization of multiple agents using decentralized navigation functions. Robotics: Science and Systems, 2005.

[21] M.M. Zavlanos and K.J. Kyriakopoulos. Decentralized motion control of multiple mobile agents. 11th Mediterranean Conference on Control and Automation, 2003. 